\title{
A VISUAL SENSITIVITY MODEL BASED STEREO IMAGE WATERMARKING SCHEME
}

\author{
Yaqing Niu, Wided Souidene and Azeddine Beghdadi \\ L2TI, University Paris 13, France
}

\begin{abstract}
We present a visual sensitivity model based method for watermarking high definition (HD) stereo images in DCT domain. Our proposal is devoted to make the stereo image watermarking scheme as robust and invisible as possible. Visual sensitivity refers to the ability of human observers to detect distortion in visual field. In order to achieve the best tradeoff point of robustness and transparency of the watermarked images, the watermarking scheme is based on the visual sensitivity model to determine the perceptual adjustment on watermark insertion. The performance of the proposed method has been tested under various attacks producing very promising robustness results while retaining the watermark transparency.
\end{abstract}

Index Terms - Just Noticeable Distortion, Stereo Image Watermarking, Visual Sensitivity Model

\section{INTRODUCTION}

Stereo images are image pairs acquired from two slightly different perspectives. One can perceive stereo images in three dimension when each image of a stereo pair is viewed by its respective eye of the viewer. Therefore, stereo images provide us a way to represent three dimensional scene using two dimensional image pairs. Stereo image pairs have been used in many applications such as flight simulator for pilot training, medical surgery, virtual reality games, robot vision, military surveillance, and autonomous navigation. With the increase of using stereo images, there is a strong necessity for developing watermarking techniques, which protect the intellectual property copyright of the original stereo images.

Digital watermarking has been extensively studied for two dimensional images but very few algorithms have been proposed for the watermarking of stereo images. A reversible watermarking method on stereo embedding is presented in [1]. This method recovers the missing image by using the difference between the two stereo images together with the embedded image. By embedding into one frame of the stereo pair the information needed to recover the other frame, the transmission and storage requirements are halved. The objective of this method is not for copyright protection but for storage and bandwidth reduction. Similar idea is proposed in [2]. In this paper, a digital watermarking algorithm in discrete wavelet transform (DWT) domain for stereo image coding is presented. A disparity-image is computed from the pair of stereo images using a frequency domain based matching criteria. This disparity-image is used as a watermark and embedded into the degraded host (left stereo) image. On the receiver's end, disparity-image is extracted and can be used to recover right image of stereo pair as well as recovering the 3D information of scene. In [3] a reversible watermarking method where the embedding is performed in the three color components of one frame of a stereo image. By embedding into the three color channels of a stereo frame the information needed to recover the other frame, the transmission/storage requirements are halved. The goal of this proposed method is for storage and bandwidth reduction as well. A semifragile stereo image watermarking scheme in the wavelet domain is proposed in [4]. The watermarking method is robust toward JPEG compression while fragile with respect to intentional attacks such as rotation and rows/columns removal.

In this paper, we propose a visual sensitivity model guided stereo image watermarking scheme in the DCT domain. We focus on solving the problem of how to achieve the embedded watermark of stereo images in a more robust and invisible manner. No previous efforts towards achieving the best tradeoff point of robustness and transparency in stereo image watermarking are found in the literature. Visual sensitivity refers to the ability of human observers to detect distortion in visual field. In order to achieve the best trade-off point of robustness and transparency of the watermarked images, the watermarking scheme is based on the visual sensitivity model to determine the perceptual adjustment on watermark insertion. The performance of the proposed method has been tested under various attacks producing very promising robustness results while retaining the watermark transparency.

This paper is organized as follows. In Section 2, the visual sensitivity model guided stereo image watermarking scheme is presented. The proposed scheme is tested against common attacks, such as additive Gaussian noise, JPEG compression, Gaussian lowpass filtering and valumetric scaling in Section 3. Finally, the paper is concluded in Section 4. 


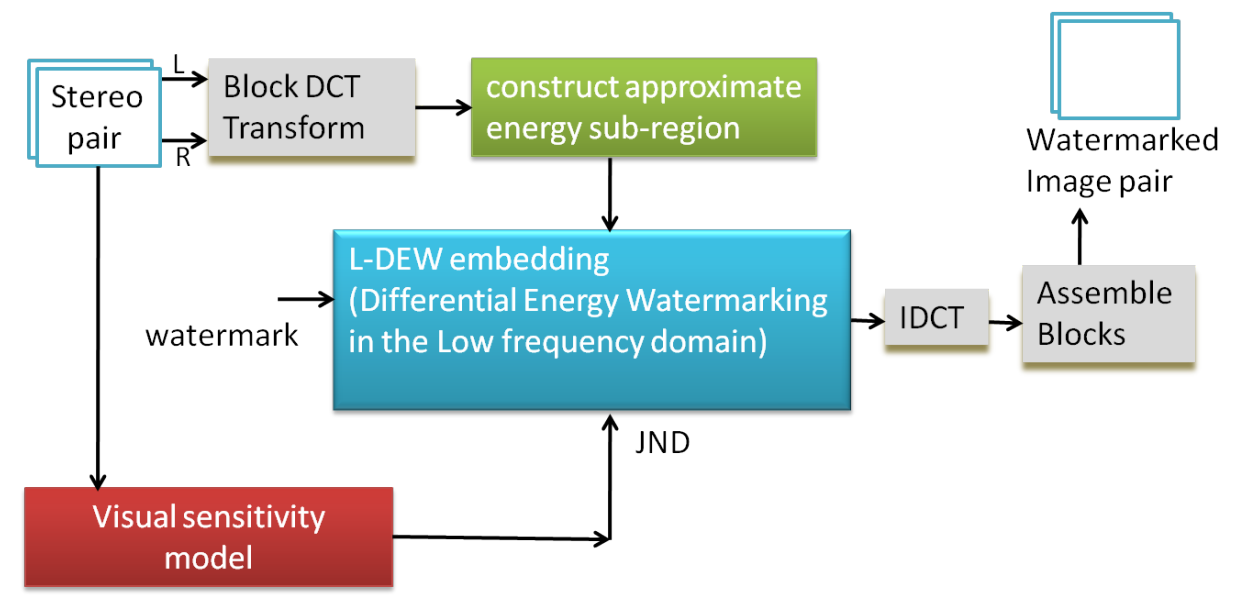

Fig. 1. Block diagram of the proposed scheme

\section{THE VISUAL SENSITIVITY MODEL GUIDED STEREO IMAGE WATERMARKING SCHEME}

In the following, we discuss an effective visual sensitivity model guided stereo image watermarking scheme in the DCT domain. The watermarking scheme, where visual sensitivity model are fully used to determine scene-adaptive upper bounds on watermark insertion, allows us to provide more strength transparent watermark. We exploit the visual sensitivity model guided stereo image watermarking scheme to embed watermark as shown in Fig. 1.

\subsection{The visual sensitivity model}

Human visual system (HVS) makes final evaluations on the quality of images that are processed and displayed. For a well-designed watermark approach, in order to maintain the image quality and at the same time increase the probability of the watermark detection, it is necessary to take the HVS into consideration. Visual sensitivity refers to the ability of human observers to detect distortion in visual field. Numerically, visual sensitivity can be regarded as the inverse of the just noticeable distortion (JND), which refers to the maximum distortion thresholds in pixels or subbands that the HVS does not perceive. JND is an efficient model to represent the perceptual redundancies. Here we compute the JND estimation [5][6] which incorporates Spatial CSF, luminance adaptation and contrast masking. The first factor Spatial CSF describes the effect of spatial frequency on the human visual sensitivity. Human eyes show a band-pass property in the spatial frequency domain. That means the HVS are more sensitive to middle spatial frequency. The second factor is luminance adaptation. The HVS is more sensitive to the noise in medium gray regions, so the visibility threshold is higher in very dark or very light regions. Because our base threshold is detected at the middle intensity value, for other intensity values, a modification factor needs to be included. This effect is called the luminance adaptation effect. The third factor is the contrast masking effect. Contrast masking refers to the reduction in the visibility of one visual component in the presence of another one. The overall JND given in Eq. 1 can be determined by the base threshold $T_{B A S E s}$, the luminance adaptation factor $a_{\text {Lum }}$ and the contrast masking factor $a_{\text {Contrast }}$ as follows:

$T_{J N D}(n, i, j)=T_{B A S E s}(n, i, j) * a_{\text {Lum }}(n) * a_{\text {Contrast }}(n, i, j)$

$T_{J N D}$ is the JND estimator to guide watermarking for digital stereo images, where $n$ is the index of a block in the image, $i$ and $j$ are the DCT coefficients indices.

\subsection{The visual sensitivity model guided stereo image wa- termarking}

The scheme first constructs a set of approximate energy subregions using the Improved Longest Processing Time (ILPT) algorithm [7], and then enforces an energy difference between every two sub-regions to embed watermarking bits [8] [9] under the control of visual sensitivity model [5][6].

\subsubsection{Watermark embedding}

Firstly we decompose the original stereo image pairs into non-overlapping $8 \times 8$ blocks and compute the energy of the low-frequency DCT coefficients in the zigzag sequence (with $i+j \leq 5$ ). Secondly, we use the visual sensitivity model briefly described in Section 2.1 to calculate the perceptual visibility threshold profile for DCT coefficients. The watermark bit string is embedded bit-by-bit in a set of regions of the original image pairs. Each region is divided into two sub-regions as shown in Fig. 2 . A single bit is embedded by modifying the energy of two sub-regions separately. For better imperceptibility, approximate energy sub-regions has to be constructed. Thus, at last we obtain approximate energy 


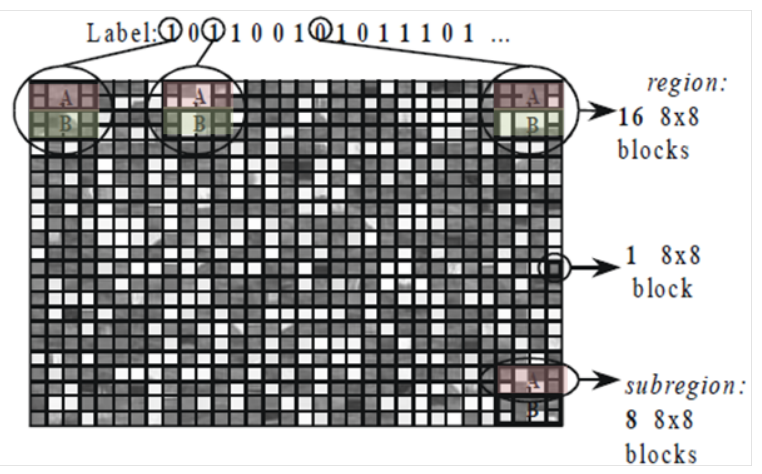

Fig. 2. Watermark bit corresponding to approximate energy sub-regions

sub-regions where we can embed the watermark by modifying the energy of two sub-regions. Each bit of the watermark bit string is embedded in its constructed bit-carrying-region. For instance, in Fig. 2 each bit is embedded in a region of 16 DCT blocks. The value of the bit is encoded by introducing an energy difference between the low frequency DCT coefficients of the top half of the region (denoted by sub-region A) containing in this case 8 DCT blocks, and the bottom half (denoted by sub-region B) also containing 8 DCT blocks. If the watermark to be embedded is 1 , the energy of subregion A should be increased and the energy of sub-region $B$ should be decreased. If the watermark to be embedded is 0 , the energy of sub-region A should be decreased and the energy of sub-region B should be increased. The energy of each sub-region is modified by adjusting their low-frequency DCT coefficients accordingly under the control of the visual sensitivity model in Section 2.1 as Eq. 2, where $C(n, i, j)$ and $C(n, i, j)^{m}$ are the DCT coefficient before and after watermark insertion, $\operatorname{Sign}($.$) is the sign function, P M$ is positive modulation which means increase the energy and $N M$ is negative modulation which means decrease the energy, $T_{J N D}(n, i, j)$ is the perceptual visibility threshold by JND model and $f($.$) can be expressed by Eq. 3$.

$$
\begin{aligned}
& C(n, i, j)^{m} \\
& =\left\{\begin{array}{l}
C(n, i, j)+ \\
\operatorname{sign}(C(n, i, j)) * f\left(C(n, i, j), T_{J N D}(n, i, j)\right), \quad P M \\
C(n, i, j)- \\
\operatorname{sign}(C(n, i, j)) * f\left(C(n, i, j), T_{J N D}(n, i, j)\right), \quad N M
\end{array}\right.
\end{aligned}
$$

$$
\begin{aligned}
& f\left(C(n, i, j), T_{J N D}(n, i, j)\right) \\
& \quad= \begin{cases}0, & C(n, i, j)<T_{J N D}(n, i, j) \\
T_{J N D}(n, i, j), & C(n, i, j) \geq T_{J N D}(n, i, j)\end{cases}
\end{aligned}
$$

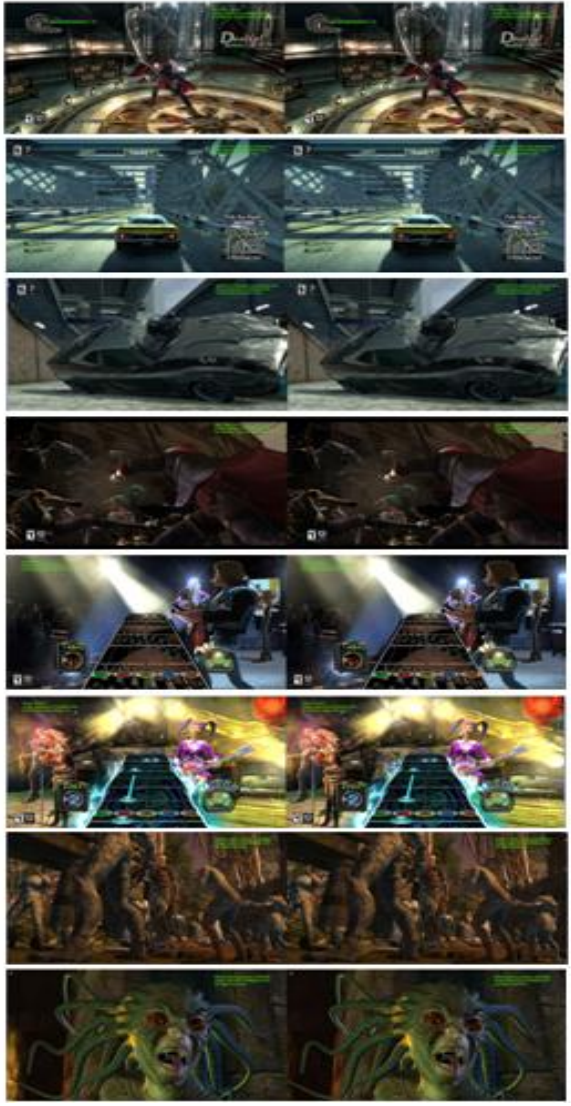

Fig. 3. Eight test stereo images for the experiments

\subsubsection{Watermark extraction}

Firstly we decompose the watermark embedded stereo image pairs into non-overlapping $8 \times 8$ blocks and compute the energy of the low-frequency DCT coefficients. Secondly, we calculate energy of each sub-region. At last, we compare the energy of sub-region A with sub-region B. If the energy of sub-region $\mathrm{A}$ is higher than the energy of sub-region $\mathrm{B}$, the watermark embedded is 1 . If the energy of sub-region $A$ is lower than the energy of sub-region B, the watermark embedded is 0 . The watermark is extracted accordingly.

\section{EXPERIMENTAL RESULTS AND PERFORMANCE ANALYSIS}

We construct a series of tests to observe the performance of the visual sensitivity model guided stereo image watermarking scheme in terms of watermark's visual quality and robustness. Eight 1080 x 1920 HD stereo images are chosen as test images for this experiment (shown in Fig. 3 with different visual contents). The original watermark is a binary image of 


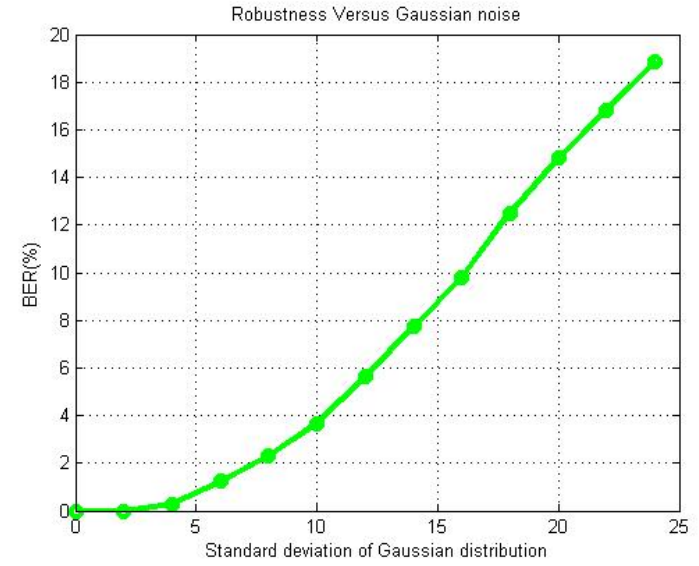

Fig. 4. Robustness versus Gaussian noise

"logo" with size 50 x 81 .

\subsection{Visual quality}

In order to test the visual quality of the watermarked stereo images, we conduct the subjective viewing tests in a room illuminated by fluorescent ceiling lights, and these are typical conditions under which people would view digital images. Ten viewers (five are experts in image processing field and five are novice observers) are involved in experiments. The subjects ages are $21-50$, and their eyesight was either normal or had been corrected to be normal with spectacles. The tests were conducted with NVIDIA GeForce 3D Vision Kit and true $120 \mathrm{~Hz}$ LCD monitors. The viewing distance was three times the hight of the stereo images. Eight stereo images presented in Fig. 3 are used for experiments. In each session of the experiment, subjects viewed original stereo image pair and watermarked stereo image pair. The order of the presentation of the stereo image pair was randomized in each session. The subjects were asked to respond whether they felt quality degradation. From the subjective viewing tests we find the watermark hardly noticeable in the resultant watermarked image pair which showed the model yield values no larger than the actual HVS thresholds.

\subsection{Robustness}

We present robustness results with different attacks like Gaussian noise, JPEG compression, Gaussian lowpass filtering and valumetric scaling. Eight stereo images presented in Fig. 3 are used for the robustness tests. Averaged watermark robustness results of the eight test stereo images are shown in Fig. 4, Fig. 5, Fig. 6 and Fig. 7. For each category of distortion, the watermarked image pair were modified with a varying magnitude of distortion and the Bit Error Rate (BER) of the extracted watermark was then computed.

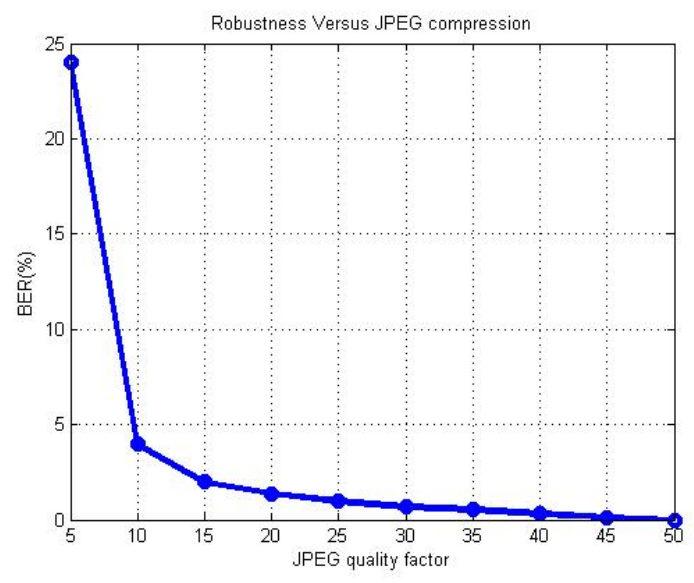

Fig. 5. Robustness versus JPEG Compression

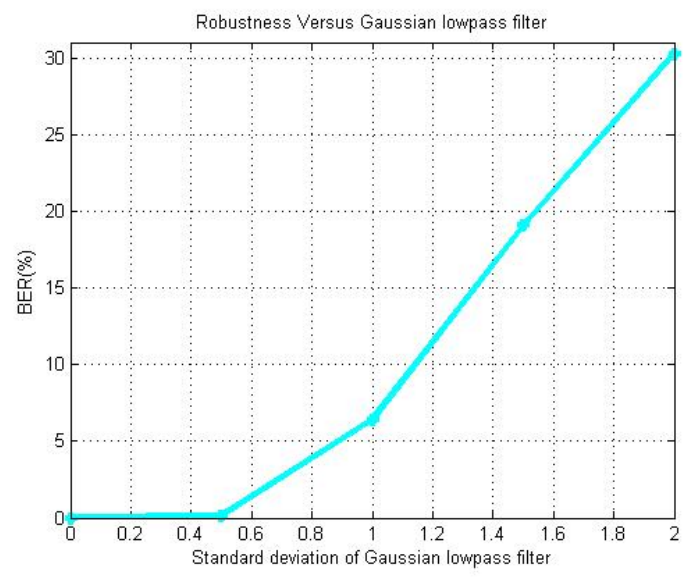

Fig. 6. Robustness versus Gaussian lowpass filtering

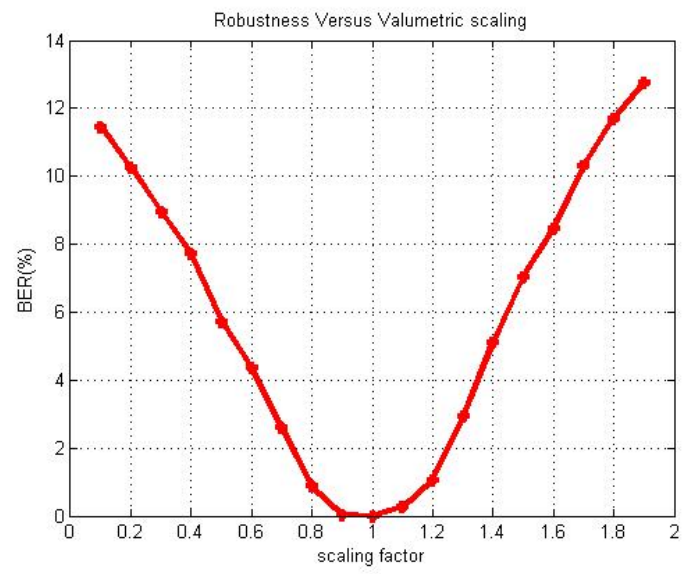

Fig. 7. Robustness versus Valumetric Scaling 
The normal distributed noise with mean 0 and standard deviation $\sigma$ is added to the watermarked image pair, where $\sigma$ varies from 0 to 24 . The experimental results are presented in Fig. 4. Following the practice, we considered the watermarking scheme to be robust if at least $80 \%$ of the watermarks were correctly retrieved, i.e. the BER is below $20 \%$. If we establish a threshold at a bit error rate of $20 \%$, the visual sensitivity model guided stereo image watermarking scheme is robust to standard deviations up to 24 .

We test the watermark robustness versus JPEG compression. The watermarked image pairs are compressed with different quality factor varying from 5 to 50 . After decompression and watermark extraction, the watermark bit errors introduced by compression are shown in Fig. 5. We can see that if the JPEG quality factor is decreased to 10 , there will be only about $3.96 \%$ bit error rate based on the visual sensitivity model guided stereo image watermarking scheme.

The watermarked frames were filtered with Gaussian lowpass filters of standard deviation $\sigma$ varying from 0 to 2 . The experiment was repeated for different values of $\sigma$ and the bit error rate was computed. Fig. 6 summarizes the experimental results. It shows the visual sensitivity model guided stereo image watermarking scheme can resist Gaussian lowpass filter of standard deviation $\sigma$ up to 1.5 as BER is less than $20 \%$.

Valumetric scaling is another simple but important distortion. The experiment shown in Fig. 7 reduced the intensities as scaling factor varied from 1 to 0.1 , and increased the intensities as scaling factor varied from 1 to 2 . Fig. 7 demonstrates that the visual sensitivity model guided stereo image watermarking scheme's performance against valumetric scaling. It shows the visual sensitivity model guided stereo image watermarking scheme can resist valumetric scaling of scaling factor vary from 0.1 to 2 as BER is less than $20 \%$.

\section{CONCLUSIONS}

We present a visual sensitivity model based method for watermarking high definition (HD) stereo images in DCT domain. Our proposal is devoted to make the stereo image watermarking scheme as robust and invisible as possible. No previous efforts towards achieving the best trade-off point of robustness and transparency in stereo image watermarking are found in the literature. Visual sensitivity refers to the ability of human observers to detect distortion in visual field. In order to achieve the best trade-off point of robustness and transparency of the watermarked images, the watermarking scheme is based on the visual sensitivity model to determine the perceptual adjustment on watermark insertion. The performance of the proposed method has been tested under various attacks producing very promising robustness results while retaining the watermark transparency. Ongoing and future developments focus on combining stereoscopic visual saliency to increase the watermark robustness and transparency.

\section{ACKNOWLEDGMENTS}

This work has been financially supported by the DGCIS France in the framework of the $H D 3 D^{2}$ project of the Cap Digital competitiveness cluster.

\section{REFERENCES}

[1] D. Coltuc and I. Caciula, "Stereo embedding by reversible watermarking: Further results," in International Symposium on Signals, Circuits and Systems (ISSCS). IEEE, 2009, pp. $1-4$.

[2] S. Kumar, B. Raman, and M. Thakur, "Real coded genetic algorithm based stereo image watermarking," International Journal of Secure Digital Information Age, vol. 1, pp. 23-33, June 2009.

[3] D. Coltuc, I. Caciula, and H. Coanda, "Color stereo embedding by reversible watermarking," in $3 r d$ International Symposium on Electrical and Electronics Engineering (ISEEE). IEEE, 2010, pp. $256-259$.

[4] P. Campisi, "Object-oriented stereo-image digital watermarking," Journal of Electronic Imaging, vol. 17, Oct 2008.

[5] Y.Q. Niu, J.B. Liu, S. Krishnan, and Q. Zhang, "Combined just noticeable difference model guided image watermarking," in IEEE Int. Conf., Multimedia and Expo 2010 workshops. IEEE, 2010.

[6] Y.Q. Niu, M. Kyan, S. Krishnan, and Q. Zhang, "A combined just noticeable distortion model guided image watermarking," Signal, Image and Video Processing, 2010.

[7] F. H. Zou, "Research of robust video watermarking algorithms and related techniques," A Dissertation Submitted for the Degree of Doctor of Philosophy in Engineering, 2006.

[8] H.F. Ling, Z.D. Lu, and F.H. Zou, "Improved differential energy watermarking (idew) algorithm for dct-encoded imaged and video," in the Seventh International Conference on Signal Processing (ICSP'2004). IEEE, 2004, pp. 2326-2329.

[9] G.C. Langelaar and R.L. Lagendijk, "Optimal differential energy watermarking of dct encoded images and video," IEEE Trans. Image Process, vol. 10, pp. 148-158, 2001. 